\title{
Further Extensions of a Legendre Function Integral
}

\author{
By Henry E. Fettis*
}

Abstract. The integral

$$
\int_{z}^{1}\left(\frac{1-t}{2}\right)^{\beta-1}\left(\frac{1-t}{1+t}\right)^{\mu / 2} \ln \left(\frac{1-t}{2}\right) P_{\nu-1}^{\mu}(t) d t
$$

is evaluated as a hypergeometric function for arbitrary values of " $\nu ", " \mu$ ", $-1 \leqslant z \leqslant 1$, and $\operatorname{Re}(\beta)>0$.

In this paper, we obtain expressions for integrals of the type

$$
\int_{z}^{1}\left(\frac{1-t}{2}\right)^{\beta-1}\left(\frac{1-t}{1+t}\right)^{\mu / 2} \ln \left(\frac{1-t}{2}\right) P_{\nu}^{\mu}(t) d t
$$

where $P_{\nu}^{\mu}(z)$ is the Legendre function, $-1 \leqslant z \leqslant 1$, and $\operatorname{Re}(\beta)>0$. The relations thus obtained include as special cases results given previously by Blue [1], Gautschi [2], Ainsworth and Liu [3], [6], Gatteschi [7], and the present author [4].

The starting point of the development is the beta-transform for the hypergeometric function ${ }_{p} F_{q}(z)$ as given in [5, Art. 5.2.3]:

(2) $\left.\frac{\Gamma(\beta+\gamma)}{\Gamma(\beta) \Gamma(\gamma)} \int_{0}^{1} t^{\beta-1}(1-t)^{\gamma-1}{ }_{p} F_{q}\left(\begin{array}{c}\alpha_{p} \\ \rho_{q}\end{array} \mid \zeta t\right) d t={ }_{p+1} F_{q+1}\left(\begin{array}{c}\beta, \alpha_{p} \\ \beta+\gamma, \rho_{q}\end{array}\right) \zeta\right)$

which, with an obvious change of variables, may be written

$$
\begin{aligned}
& \frac{\Gamma(\beta+\gamma)}{\Gamma(\beta) \Gamma(\gamma)} \int_{z}^{1}(1-x)^{\beta-1}(x-z)_{p}^{\gamma-1} F_{q}\left(\begin{array}{c}
\alpha_{p} \\
\rho_{q}
\end{array} \mid \frac{1-x}{2}\right) d x \\
& =(1-z)_{p+1}^{\beta+\gamma-1} F_{q+1}\left(\begin{array}{c}
\beta, \alpha_{p} \\
\beta+\gamma, \rho_{q}
\end{array} \mid \frac{1-z}{2}\right),
\end{aligned}
$$

and since

$$
\Gamma(1-\mu) P_{\nu-1}^{\mu}(x)=\left(\frac{1+x}{1-x}\right)^{\mu / 2}{ }_{2} F_{1}\left(\begin{array}{c}
\nu, 1-\nu \\
1-\mu
\end{array} \mid \frac{1-x}{2}\right)
$$

Received July 25, 1983; revised April 3, 1984 and August 14, 1984.

1980 Mathematics Subject Classification. Primary 33A65, 33A35; Secondary 33A30, 33A15.

Key words and phrases. Jacobi polynomials, Legendre functions, hypergeometric functions, $\Gamma$-functions, definite integrals, beta transform.

*Deceased December 15, 1984. 
(3) gives

$$
\begin{aligned}
\Gamma(1 & -\mu) \int_{z}^{1}\left(\frac{1-x}{2}\right)^{\beta-1}\left(\frac{x-z}{2}\right)^{\gamma-1}\left(\frac{1-x}{1+x}\right)^{\mu / 2} P_{\nu-1}^{\mu}(x) d x \\
& =2\left(\frac{1-z}{2}\right)^{\beta+\gamma-1} \frac{\Gamma(\beta) \Gamma(\gamma)}{\Gamma(\beta+\gamma)}{ }_{3} F_{2}\left(\begin{array}{c}
\beta, \nu, 1-\nu \\
\beta+\gamma, 1-\mu
\end{array} \mid \frac{1-z}{2}\right) .
\end{aligned}
$$

Equation (5) may be specialized in a number of ways. For example, if $\beta=1$, we get

$$
\begin{gathered}
\Gamma(1-\mu) \int_{z}^{1}\left(\frac{x-z}{2}\right)^{\gamma-1}\left(\frac{1-x}{1+x}\right)^{\mu / 2} P_{\nu-1}^{\mu}(x) d x \\
=\frac{2}{\gamma}\left(\frac{1-z}{2}\right)^{\gamma}{ }_{3} F_{2}\left(\begin{array}{c}
1, \nu, 1-\nu \\
1+\gamma, 1-\mu
\end{array} \mid \frac{1-z}{2}\right) ; \\
\Gamma(1-\mu) \int_{-1}^{1}\left(\frac{1+x}{2}\right)^{\gamma-1}\left(\frac{1-x}{1+x}\right)^{\mu / 2} P_{\nu-1}^{\mu}(x) d x \\
=\frac{2}{\gamma}{ }_{3} F_{2}\left(\begin{array}{c}
1, \nu, 1-\nu \\
1+\gamma, 1-\mu
\end{array} \mid\right),
\end{gathered}
$$

and, if, in addition, $\mu=0$, the ${ }_{3} F_{2}$ function reduces to an ${ }_{2} F_{1}$, and we obtain the following:

$$
\begin{gathered}
\int_{z}^{1}\left(\frac{x-z}{2}\right)^{\gamma-1} P_{\nu-1}(x) d x=\frac{2}{\gamma}\left(\frac{1-z}{2}\right)_{2}^{\gamma} F_{1}\left(\begin{array}{c}
\nu, 1-\nu \\
1+\gamma
\end{array} \mid \frac{1-z}{2}\right) \\
\int_{-1}^{1}\left(\frac{x+1}{2}\right)^{\gamma-1} P_{\nu-1}(x) \cdot d x \\
=\frac{2}{\gamma}{ }_{2} F_{1}\left(\begin{array}{c}
\nu, 1-\nu \\
1+\mu
\end{array} \mid 1\right)=\frac{2[\Gamma(\gamma)]^{2}}{\Gamma(\gamma+\nu) \Gamma(\gamma-\nu+1)},
\end{gathered}
$$

the latter being equivalent to Eq. (3) of [2].

Similarly, by taking $\gamma=1$ in (5) we get

$$
\begin{gathered}
\Gamma(1-\mu) \int_{z}^{1}\left(\frac{1-x}{2}\right)^{\beta-1}\left(\frac{1-x}{1+x}\right)^{\mu / 2} P_{\nu-1}^{\mu}(x) d x \\
=\frac{2}{\beta}\left(\frac{1-z}{2}\right)_{3}^{\beta} F_{2}\left(\begin{array}{c}
\beta, \nu, 1-\nu \\
1+\beta, 1-\mu
\end{array} \mid \frac{1-z}{2}\right)
\end{gathered}
$$

(8b) $\Gamma(1-\mu) \int_{-1}^{1}\left(\frac{1-x}{2}\right)^{\beta-1}\left(\frac{1-x}{1+x}\right)^{\mu / 2} P_{\nu-1}^{\mu}(x) d x=\frac{2}{\beta}{ }_{3} F_{2}\left(\begin{array}{c}\beta, \nu, 1-\nu \\ 1+\beta, 1-\mu\end{array}\right)$

and it appears that, in general, (8a) and (8b) cannot be reduced or simplified except in the special case where $\mu=0, z=-1$, and $\nu$ is an integer, $n$, in which instance, the integrals in (7b) and (8b) differ only in sign, leading to the relation

$$
\frac{1}{\beta^{3}} F_{2}\left(\begin{array}{c}
\beta, n, 1-n \\
1+\beta, 1
\end{array} \mid 1\right)=\frac{(-1)^{n+1}[\Gamma(\beta)]^{2}}{\Gamma(\beta+n) \Gamma(\beta-n+1)},
$$

a result also obtainable directly from formula 5.2.4(2) of [5]. 
On the other hand, if $\nu=n$ and $\mu$ is not a positive integer, the functions $P_{n-1}^{\mu}(x)$ and $P_{n-1}^{-\mu}(x)$ differ only by a numerical factor;

$$
P_{n-1}^{-\mu}(-x)=(-1)^{n} \frac{\Gamma(n-\mu)}{\Gamma(n+\mu)} P_{n-1}^{\mu}(x)
$$

which leads to the following, and not immediately evident, relation:

$$
\begin{gathered}
(-1)^{n-1}\left[(1-\mu)_{n-1}\right]_{3} F_{2}\left(\begin{array}{c}
\beta, n, 1-n \\
1+\beta, 1-\mu
\end{array}\right) \\
=\left[(1+\mu)_{n-1}\right]_{3} F_{2}\left(\begin{array}{c}
1, n, 1-n \\
1+\beta, 1+\mu
\end{array}\right) .
\end{gathered}
$$

The corresponding integrals involving the logarithmic term are obtained by differentiation with respect to " $\beta$ " (or " $\gamma$ "). However, this can, apparently, only be done explicitly in two special cases, one being that where the integral is expressible by gamma-functions, as in ( $7 \mathrm{~b})$. In this instance, the result becomes the one given by Gautschi and the present author ([2], [4]), and previously for integer " $\nu$ " by Ainsworth and Liu [3]. (Cf. [2, Eq. (1)].) The second case where explicit expressions are obtainable for the logarithmic integrals occurs when $\gamma=1$ (Eqs. (8a), (8b)), since then we have

$$
\begin{aligned}
& -\beta^{2} \frac{\partial}{\partial \beta}\left\{\frac{1}{\beta^{3}} F_{2}\left(\begin{array}{c}
\beta, \nu, 1-\nu \\
1+\beta, 1-\mu
\end{array} \mid \frac{1-z}{2}\right)\right\} \\
& ={ }_{4} F_{3}\left(\begin{array}{c}
\beta, \beta, \nu, 1-\nu \\
1+\beta, 1+\beta, 1-\mu
\end{array} \mid \frac{1-z}{2}\right),
\end{aligned}
$$

the result being a generalization of one previously found by Ainsworth [6] for $z=-1, \mu=0$, and integer values of $\nu$ and $\beta$. Equation (12) also provides an explicit expression for the ${ }_{4} F_{3}$ function when $z=-1, \mu=0$. Further, the above idea can be extended to the case where $\gamma$ is a positive integer, $m$, by noting that expansion of the term $(1-t)^{m-1}$ in the beta-transform (2) by the binomial theorem leads to the result**

$$
\begin{aligned}
& \frac{1}{\beta_{3} F_{2}}\left(\begin{array}{c}
\beta, a, b \\
\beta+m, c
\end{array}\right) z \\
& \quad=\frac{\Gamma(\beta+m)}{\Gamma(\beta+1)} \sum_{n=0}^{m-1}(-1)^{m}\left(\begin{array}{c}
m-1 \\
n
\end{array}\right)\left(\frac{1}{\beta+n}\right){ }_{3} F_{2}\left(\begin{array}{c}
\beta+n, a, b \\
\beta+n+1, c
\end{array} \mid z\right) .
\end{aligned}
$$

Additional observations are that, although (5), (6) and (8) were originally restricted to noninteger values of " $\mu$ ", they are easily modified to include integer values of this parameter by applying the limiting expression given by Eq. 6.3(12) of [5] to the hypergeometric function in (5), and that with the aid of the relation

$$
(\sin \nu \pi) Q_{\nu-1}^{\mu}(x)=\frac{\pi}{2} \cos (\mu-\nu) \pi \frac{\Gamma(\nu+\mu)}{\Gamma(\nu-\mu)} P_{\nu-1}^{-\mu}(x)+\cos (\mu \pi) P_{\nu-1}^{\mu}(-x)
$$

\footnotetext{
** The author is indebted to the referee for these suggestions.
} 
(6b) and (8b) may be combined to give, for noninteger " $\nu$ " and " $\mu$ ", the following:

$$
\begin{aligned}
\sin (\nu \pi) & \int_{-1}^{1}\left(\frac{1-t}{2}\right)^{\beta-1}\left(\frac{1-t}{1+t}\right)^{\mu / 2} Q_{\nu-1}^{\mu}(t) d t \\
& =\frac{\pi}{\beta} \cos (\mu+\nu) \pi\left\{\frac{\Gamma(\nu+\mu)}{\Gamma(1+\mu) \Gamma(\nu-\mu)}{ }_{3} F_{2}\left(\begin{array}{c}
1, \nu, 1-\nu \\
1+\beta, 1+\mu
\end{array} \mid 1\right)\right\} \\
& +\frac{\cos (\mu \pi)}{\Gamma(1-\mu)}\left\{{ }_{3} F_{2}\left(\begin{array}{c}
\beta, \nu, 1-\nu \\
1+\beta, 1-\mu
\end{array} \mid 1\right)\right\} .
\end{aligned}
$$

Finally, it may be noted that, since

$$
P_{n}^{(p, q)}(2 z-1)=(-1)^{n} \frac{(q+1)_{n}}{n !}{ }_{2} F_{1}\left(\begin{array}{c}
-n, p+q+n+1 \\
q+1
\end{array} \mid z\right),
$$

the beta-transform (3) also provides a simple means of obtaining results relating to the Jacobi polynomials $P_{n}^{(p, q)}(z)$ which are more general than some of those given in [7].** For example, Eq. (1.3) (loc. cit.) may be generalized in this way to

$$
\begin{aligned}
& (-1)^{n} \frac{n !}{(q+1)_{n}} \int_{-1}^{z}(z-t)^{\alpha}(1+t)^{\beta+u} P_{n}^{(p, q)}(2 t-1) d t \\
& =(1+z)^{\alpha+\beta-1}{ }_{3} F_{2}\left(\begin{array}{c}
-n, p+q+n+1, \beta+u+1 \\
q+1, \alpha+\beta+u+2
\end{array} \mid \frac{1+z}{2}\right)
\end{aligned}
$$

which, in the special case where $z=1, p=\alpha, q=\beta$, can, with the aid of formula 5.2.4(2) of [5], be reduced to Eq. (1.5) of the first cited paper.

1. J. L. Blue, “A Legendre polynomial integral,” Math. Comp., v. 33, 1979, pp. 739-741.

2. W. GaUtschI, "On the preceding paper 'A Legendre polynomial integral' by James L. Blue”, Math. Comp., v. 33, 1979, pp. 742-743.

3. O. R. Ainsworth \& C. K. LiU, "An application of Legendre functions in the inversion of a Hilbert matrix,” J. Franklin Inst., v. 299, No. 4, 1975, pp. 297-299.

4. H. E. FeTtis, "Extension of a result of Ainsworth \& Liu," J. Franklin Inst., v. 316, No. 2, 1983, p. 191.

5. Y. L. Luke, Mathematical Functions and Their Approximations, Academic Press, New York, 1975.

6. O. R. AINSWORTH, private communication, 1982.

7. L. Gatteschi, “On some orthogonal polynomial integrals," Math. Comp., v. 35, 1980, pp. 1291-1298. 